\title{
ESTUDO DA PERDA DE CARGA E DA ENERGIA DE MISTURA EM PASTAS DE CIMENTO COM MICROESFERA CERÂMICA
}

\author{
Eduardo da Cunha Hora Paraiso ${ }^{1}$ \\ Cláudia Miriam Scheid ${ }^{1}$ \\ Luís Américo Calçada ${ }^{1}$ \\ Deividson Silveira dos Santos ${ }^{2}$ \\ Caroline Eulino Gonçalves Pereira ${ }^{3}$ \\ Edilson Adrião Cabral ${ }^{3}$
}

\begin{abstract}
Resumo: Diversos cenários e diferentes formações rochosas são encontrados durante a perfuração de poços exploratórios de petróleo. Durante o processo de perfuração, a etapa de cimentação é essencial, pois proporciona estabilidade ao poço, cria uma zona de isolamento, garantindo, assim, sua integridade. Em regiões com formações rochosas frágeis, utiliza-se pasta de cimento com baixa densidade. Neste trabalho, uma pasta de alta compacidade e densidade reduzida por meio da adição de microesferas cerâmicas ocas foi analisada. Foram realizados experimentos de bancada para avaliar a quebra dessas microesferas através do acompanhamento da densidade da pasta, em diferentes taxas de cisalhamento e energias de mistura. Além disso, foram avaliadas correlações que viabilizam a previsão da perda de carga durante o escoamento da pasta de cimento. A importância de ambos os testes se deve à necessidade do controle da pressão durante o bombeio da pasta no poço. Para a previsão da perda de carga foi construído um loop de escoamento que apresenta similaridade dinâmica com poços reais. Nestes testes foram avaliados modelos reológicos, correlações de fator de atrito e de diâmetro hidráulico. Os resultados dos testes de bancada mostraram uma variação significativa na densidade, proveniente da quebra das microesferas, havendo uma relação desta com a energia de mistura. No escoamento da pasta na unidade, chegou-se a um conjunto de correlações que adequadamente previram a perda de carga da pasta analisada.
\end{abstract}

Palavras-chave: poços de petróleo, cimentação, perda de carga.

Abstract: Several scenarios and different rock formations are found when drilling oil wells. During the drilling process, cementing is essential as it provides well stability, creates a zonal isolation ensuring well integrity. In regions with fragile rock formations, low-density cement slurry is used. In this research slurry of high compactness and low density through the addition of hollow ceramic microspheres was analyzed. Bench experiments were made to evaluate the breakdown of these microspheres by monitoring the slurry density at different shear rates and mixing energy. Furthermore, we evaluated correlations that enable the pressure drop prediction during cement slurry flow. The importance of both tests is due to the necessity of checking pressure during pumping the slurry in the well. In order to predict pressure drop, a flow loop with dynamic similarity to actual wells was built. In these tests, rheological models, friction factor and hydraulic diameter correlations were evaluated. The bench test results showed a significant variation in density from the microspheres breakage, having a relation between the breakage and the mixing energy. After flowing the slurry in the experimental unit, we reached a set of correlations that adequately predicted pressure drop of the analyzed slurry.

Keywords: oil wells, cementing, pressure loss.

${ }^{1}$ Departamento de Engenharia Química da Universidade Federal Rural do Rio de Janeiro. E-mail: eduardocunha@ufrrj.br

${ }^{2}$ Discente da Pós-Graduação em Engenharia Química da Universidade Federal Rural do Rio de Janeiro

${ }^{3}$ Discente do curso de Engenharia Química da Universidade Federal Rural do Rio de Janeiro. 


\section{INTRODUÇÃO}

O escoamento e o transporte de fluidos é uma área de estudo fundamental no meio industrial, não sendo exceção para a indústria de petróleo. Os fluidos são alvos de análise desde a perfuração até o consumo dos produtos derivados. $\mathrm{Na}$ etapa de perfuração, para que essa exploração se torne viável e alcance seu total potencial de produção, a etapa de cimentação, deve ser feita adequadamente.

As principais metas da cimentação são: propiciar a integridade do poço através da zona de isolamento evitando, assim, a migração de fluidos no anular; manter a estabilidade mecânica do poço; suportar os tubos de revestimento, dando sustentação a coluna e proteger o revestimento da corrosão por fluidos (Miranda, 2008).

Durante este processo ocorre a circulação de pasta de cimento em regiões circulares, interior da coluna de revestimento, e regiões anulares, entre a formação rochosa e o exterior da coluna de revestimento.

A cimentação é uma das etapas mais críticas durante a fase de perfuração. Problemas com o bombeio da pasta e com o preenchimento incorreto da coluna podem acarretar perda do poço (Thomas et al., 2001).

A escolha da pasta a ser utilizada na cimentação depende das características do poço. Fatores limitantes na determinação da massa específica e de propriedades físico-químicas das pastas de cimento são as pressões de fratura e de poros do poço.

Para garantir um controle efetivo da pressão no fundo do poço, além do controle das propriedades físicoquímicas do fluido é fundamental uma correta previsão da perda de carga deste escoamento.

Em condições onde a zona de formação é fraca, esgotada ou inconsolidada, possuindo baixa pressão de fratura, ou em situações onde se é utilizado uma extensa coluna de cimento faz-se necessário o uso de uma pasta leve, de baixa massa específica (Miranda, 2008).

Neste trabalho foi utilizada uma formulação de pasta de cimento leve, contendo microesfera cerâmica oca. As principais motivações foram avaliar: a quebra dessa microesfera frente a diferentes taxas de cisalhamento, as consequências dessa quebra sobre a massa específica e as propriedades reológicas. Avaliou-se ainda, com dados experimentais do escoamento da pasta, correlações da literatura pertinentes a previsão da perda de carga.

$\mathrm{O}$ intuito dessas avaliações é fomentar as possíveis diferenças obtidas nas propriedades da pasta durante $o$ preparo no laboratório e no campo. Estas possíveis mudanças são determinantes na previsão da pressão de bombeio. Além disto pretende-se definir um conjunto de equações que permita prever com relativa precisão a perda de carga do escoamento desta pasta.

\section{REVISÃO DE LITERATURA}

Neste item são apresentadas correlações pertinentes a previsão da perda de carga, que são: modelos reológicos, correlações de fator de atrito e correlações de diâmetro hidráulico. Em seguida, são apresentadas as equações utilizadas para a análise da energia de mistura. Outro ponto abordado é a utilização de materiais estendedores e suas aplicações.

\subsection{MODELOS REOLÓGICOS}

A reologia é definida como o estudo do comportamento deformacional e do fluxo de matéria submetido à tensão, sob determinadas condições termodinâmicas ao longo de um intervalo de tempo.

Segundo Campos et al.(2002), na prática, a reologia representa a relação entre a vazão de bombeamento (taxa de deformação) e a queda de pressão (tensão de cisalhamento), possibilitando a determinação do regime de fluxo do fluido.

Neste trabalho, foram analisados os modelos reológicos power law, 
Bingham e Herschel-Buckley, apresentados pelas Equações 1, 2 e 3, respectivamente (Bird et al., 2004).

$$
\begin{gathered}
\tau=k \gamma^{n} \\
\tau=\mu_{p} \gamma+\tau_{0} \\
\tau=k(\gamma)^{n}+\tau_{0}
\end{gathered}
$$

Sendo $k \quad o$ índice de consistência, $\gamma$ a taxa de deformação, $n$ o índice de comportamento, $\tau_{0}$ o limite de escoamento e $\mu_{\mathrm{p}}$ a viscosidade plástica.

\subsection{FATOR DE ATRITO}

Para fluidos não newtonianos, a viscosidade varia com a taxa de deformação e cada modelo apresenta uma equação específica para o cálculo do fator de atrito.

No regime laminar, o cálculo do fator de atrito para os modelos power law, Bingham e Herschel-Buckley é feito com auxílio de adimensionais, que são o número de Reynolds (modificado para cada modelo) e o número de Hedstrom (He) (Bird et al., 2004).

Para o modelo power law, o fator de atrito no regime laminar é calculado através da Equação 4.

$$
\mathrm{f}=\frac{16}{\operatorname{Re}_{\mathrm{P}}}
$$

Onde;

$$
\operatorname{Re}_{P}=\frac{\mathrm{D}\langle\mathrm{v}\rangle \rho}{k\left(\frac{8 v}{D}\right)^{\mathrm{n}-1}\left(\frac{3 \mathrm{n}+1}{4 \mathrm{n}}\right)^{\mathrm{n}}}
$$

Para o modelo de Bingham, o fator de atrito no regime laminar é obtido através da Equação 6.

$$
f=\frac{16}{\operatorname{Re}_{B}}\left[1+\frac{\mathrm{He}_{\mathrm{B}}}{6 \operatorname{Re}_{\mathrm{B}}}-\frac{\mathrm{He}_{\mathrm{B}}^{4}}{3 \mathrm{f}^{3} \operatorname{Re}_{\mathrm{B}}^{7}}\right]
$$

Onde;

$$
\begin{gathered}
\operatorname{Re}_{\mathrm{B}}=\frac{\mathrm{D}\langle\mathrm{v}\rangle \rho}{\mathrm{VP}_{\mathrm{B}}} \\
\mathrm{He}_{\mathrm{B}}=\frac{\mathrm{D}^{2} \rho L E_{B}}{\mathrm{VP}_{\mathrm{B}}^{2}}
\end{gathered}
$$

Para o modelo de HerschelBuckley, que possui parâmetros com características tanto do modelo power law quanto Bingham, o fator de atrito é calculado através da Equação 9.

$$
\mathrm{f}=\frac{2 \mathrm{He}_{\mathrm{HB}}}{\mathrm{Re}_{\mathrm{HB}}^{2}}+\frac{16}{\mathrm{Re}_{\mathrm{HB}}}[\mathrm{A}]^{-\mathrm{n}_{\mathrm{HB}}}
$$

Onde;

$$
\begin{gathered}
{[A]\left[\begin{array}{c}
\left.\left(1-\frac{L E_{\mathrm{HB}}}{\tau_{\mathrm{w}}}\right)^{3}+\frac{2\left(3 \mathrm{n}_{\mathrm{HB}}+1\right)}{\left(2 \mathrm{n}_{\mathrm{HB}}+1\right)}\left(\frac{\mathrm{LE}_{\mathrm{HB}}}{\tau_{\mathrm{w}}}\right)\left(1-\frac{\mathrm{LE}_{\mathrm{HB}}}{\tau_{\mathrm{w}}}\right)^{2}+\right] \\
\frac{\left(3 \mathrm{n}_{\mathrm{HB}}+1\right)}{\left(\mathrm{n}_{\mathrm{HB}}+1\right)}\left(\frac{\mathrm{LE}_{\mathrm{HB}}}{\tau_{\mathrm{w}}}\right)^{2}\left(1-\frac{\mathrm{LE} \mathrm{H}_{\mathrm{HB}}}{\tau_{\mathrm{w}}}\right)
\end{array}\right]} \\
\operatorname{Re}_{\mathrm{HB}}=\frac{8 \mathrm{D}^{\mathrm{n}_{\mathrm{HB}}}\langle\mathrm{v}\rangle^{2-\mathrm{n}_{\mathrm{HB}}} \rho}{\mathrm{k}_{\mathrm{HB}}\left[2\left(3 \mathrm{n}_{\mathrm{HB}}+1\right) / \mathrm{n}_{\mathrm{HB}}\right]_{\mathrm{HB}}^{\mathrm{h}_{\mathrm{HB}}}} \\
\frac{\mathrm{LE}_{\mathrm{HB}}}{\tau_{\mathrm{w}}}=2 \frac{\mathrm{He}_{\mathrm{HB}}}{\mathrm{f} \mathrm{Re}_{\mathrm{HB}}^{2}}
\end{gathered}
$$

Para o regime turbulento, alguns autores propuseram correlações para o cálculo do fator de atrito de fluidos não newtonianos.

Tomita (1959), avaliando o escoamento de fluidos de Bingham, propôs a Equação 13 para o cálculo do fator de atrito.

$$
\mathrm{f}=\lambda / 4
$$

Onde; 


$$
\frac{1}{\sqrt{\lambda}}=2 \log \left(\operatorname{Re} \frac{\sqrt{\lambda}}{2}\right)-0,2
$$

Através da combinação de equações presentes na literatura, Churchill (1977) propôs a Equação 15 para o cálculo do fator de atrito no regime laminar e turbulento.

$$
\mathrm{f}=2\left[\left(\frac{8}{\operatorname{Re}}\right)^{12}+\frac{1}{(\mathrm{~A}+\mathrm{B})^{3 / 2}}\right]^{\frac{1}{12}}
$$

Onde;

$$
A=\left[2,457 \ln \frac{1}{\left(\frac{7}{\operatorname{Re}}\right)^{0,9}+0,27 \frac{\varepsilon}{D}}\right]^{16}
$$

Ellis e George (1977) propuseram a Equação 17 para o cálculo do fator de atrito no regime turbulento.

$$
f=0,00454+0,645 \mathrm{Re}^{-0,70}
$$

Darby e Melson (1981) propuseram uma correlação para o cálculo do fator de atrito de fluidos de Bingham, Equação 18, a qual atende tanto o regime laminar quanto $\mathrm{o}$ turbulento.

$$
f=\left(f_{L}^{m}+f_{T}^{m}\right)^{m}
$$

Onde;

$$
\begin{gathered}
\mathrm{f}_{\mathrm{L}}=\frac{16}{\operatorname{Re}}\left[1+\frac{\mathrm{He}}{6 \mathrm{Re}}-\frac{\mathrm{He}^{4}}{3 \mathrm{f}_{\mathrm{L}}^{3} \mathrm{Re}^{7}}\right] \\
\mathrm{f}_{\mathrm{T}}=\frac{10^{\mathrm{a}}}{\operatorname{Re}^{0,193}} \\
\mathrm{~m}=1,7+\frac{40000}{\mathrm{Re}}
\end{gathered}
$$

$$
\mathrm{a}=-1,378\left[1+0,146 \mathrm{exp}\left(-2,9 \times 10^{-5} \mathrm{He}\right)\right]
$$

Darby et al. (1992) propuseram uma modificação no parâmetro (a) da Equação 18, obtendo a Equação 23 para o cálculo deste parâmetro.

$$
\mathrm{a}=-1,47\left[1+0,146 \exp \left(-2,9 \times 10^{-5} \mathrm{He}\right)\right]
$$

Com os dados de fator de atrito obtidos por Dodge e Metzner, Ostwald de Waele e Frank Schuh, Gomes (1987) propôs correlações para o cálculo do fator de atrito de forma explicita, Equações de 24 a 26.

$$
\begin{aligned}
& \mathrm{f}=0,060 \mathrm{n}^{0,462} \mathrm{Re}^{-0,223} \\
& \mathrm{f}=0,069 \mathrm{n}^{0,666} \mathrm{Re}^{-0,235} \\
& \mathrm{f}=0,110 \mathrm{n}^{0,616} \mathrm{Re}^{-0,287}
\end{aligned}
$$

\subsection{DIÂMETRO HIDRÁULICO}

As correlações de diâmetro hidráulico geram fatores geométricos capazes de representar o canal anular como um duto circular. Bourgoyne et al. (1991) apresentam duas correlações. A Equação 27, conhecida como slot, é válida quando a razão entre os diâmetros interno e externo da região anular é maior que 0,3 .

$$
D H_{1}=0,816\left(D_{2}-D_{1}\right)
$$

A segunda correlação apresentada foi desenvolvida analiticamente usando a teoria de raio hidráulico, Equação 28.

$$
D H_{2}=4 R_{H}=\left(D_{2}-D_{1}\right)
$$

Uma terceira correlação testada foi a Equação 29. Esta equação, proposta por Paraiso (2011), é baseada em dados experimentais do escoamento de fluidos em dutos anulares. 


$$
D H_{3}=0,702\left(D_{2}-D_{1}\right)+0,0014
$$

Nas equações de 27 a $29, \mathrm{D}_{2}$ e $\mathrm{D}_{1}$ são, respectivamente, o diâmetro interno do tubo externo e o diâmetro externo do tubo interno.

\subsection{ENERGIA DE MISTURA}

Segundo Nelson (1990), as propriedades físico-químicas das pastas de cimento sofrem variações em função da energia de mistura fornecida. Isto pode ser observado através da variação de viscosidade plástica da pasta de cimento, mostrada na Figura 1.

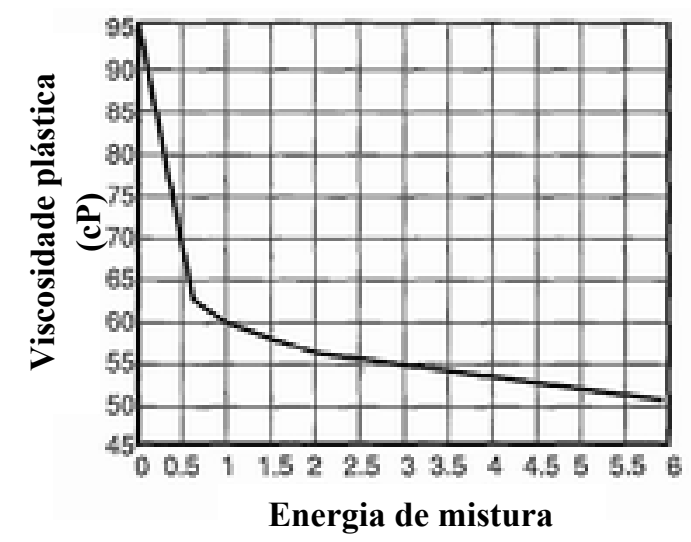

Figura 1. Viscosidade plâstica em função da energia de mistura da pasta de cimento (adaptada de Nelson, 1990).

A energia de mistura por unidade de massa de pasta pode ser obtida pela Equação 30.

$$
\frac{E}{M}=\frac{\left(K \omega^{2} t\right)}{V}
$$

Onde: $\mathrm{M}$ é a massa da pasta $(\mathrm{kg}), \mathrm{V}$ é o volume de pasta $\left(\mathrm{m}^{3}\right)$, E é a energia de mistura $(\mathrm{kJ}), \omega$ é a velocidade de rotação (Radianos/s), t é o tempo de mistura (s) e $\mathrm{K}$ é uma constante de proporcionalidade específica de cada equipamento.

Para a Equação 30, o trabalho mecânico fornecido pelo misturador durante o tempo, t, é calculado através da Equação 31 (Campos et al., 2002).

$$
E=T \omega t
$$

O torque $\mathrm{T}$ é calculado por meio da Equação 32.

$$
T=K \rho \omega
$$

Onde: $\rho$ é a densidade da pasta $\left(\mathrm{kg} / \mathrm{m}^{3}\right)$ e o valor de $\mathrm{K}$, para o misturador Chandler modelo 30-60, é $6,1 \times 10-8 \mathrm{~m}^{5} / \mathrm{s}$.

\subsection{ESTENDEDOR (REDUTOR DE MASSA ESPECÍFICA)}

A utilização das microesferas ocas como estendedores são frequentes em pastas de cimento. Segundo Nelson (1990), as microesferas possuem boa força de compressão, estabilidade térmica e propriedades isolantes. As microesferas são preenchidas com gás, o que leva a uma baixa massa específica que normalmente é entre 0,4 e $0,6 \mathrm{~g} /$ $\mathrm{cm}^{3}$. Isso permite a formulação de uma pasta de cimento estendida (de baixa massa específica), de alta força e baixa permeabilidade.

A redução da massa específica causa uma diminuição da carga hidrostática do fluido, favorecendo seu bombeamento no poço. Isto permite sua aplicação em zonas fragilizadas onde um elevado diferencial de pressão causaria um colapso da coluna.

Um cuidado adicional que se deve ter com a aplicação desse tipo de estendedor é a quebra da partícula.

Segundo Miranda (2008), as microesferas ocas são suscetíveis à quebra ao serem expostas a uma alta pressão e a elevadas taxas de cisalhamento. Este fato é comprovado nas micrografias apresentadas na Figura 2.

Nas micrografias da Figura 2b, é possível perceber que há uma quebra mais significativa quando comparado com a Figura 2a que foi exposta a um cisalhamento menos intenso. 


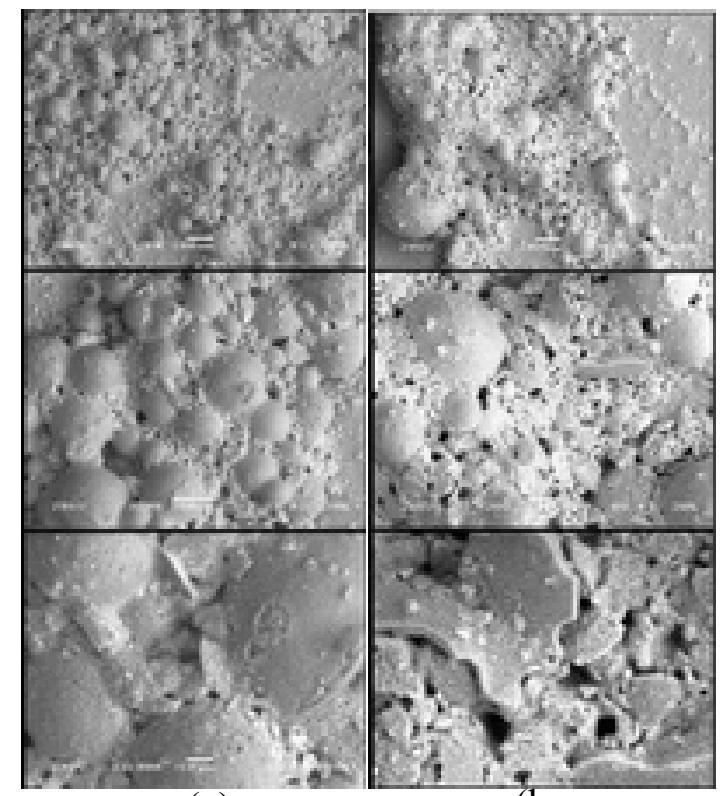

(a)

(b

Figura 2. Micrografias com aumentos de 100, 300 e 1000 vezes: (a) $3000 \mathrm{rpm}$ por $9 \mathrm{~min} 50 \mathrm{~s}$; (b) $4000 \mathrm{rpm}$ por $15 \mathrm{~s}+3000$ rpm por $9 \min 22 \mathrm{~s}+12000 \mathrm{rpm}$ por 1 min, adaptadas de Miranda (2008).

As diferenças observadas no grau de quebra das microesferas apresentadas na Figura 2 levam a pasta de cimento a valores de densidade diferentes. Este fato trás uma preocupação importante sobre a relação entre a produção das pastas em laboratório e nas sondas de perfuração.

De uma forma geral, pode-se dimensionar ou formular uma pasta com propriedades diferentes da obtida em sua produção de campo, o que implicará num mau dimensionamento da pressão de bombeio e um risco a estabilidade hidráulica e mecânica do poço.

\section{MATERIAIS E MÉTODOS}

Para a análise do escoamento da pasta de cimento foi construída uma unidade de simulação de escoamento de fluidos capaz de fornecer dados de pressão, temperatura e vazão. Para o estudo do comportamento da pasta de cimento frente à energia de mistura foram realizados testes de bancada em misturador Chandler, modelo 30-60.

\subsection{FORMULAÇÃO DA PASTA DE CIMENTO}

A pasta de cimento utilizada nessa pesquisa tem os seguintes compostos:

Materiais sólidos: cimento classe $\mathrm{G}$, fornecido pela empresa Holcim, microesfera oca de cerâmica, fornecida pela empresa $R \& D$ International e sílica ativa, fornecida pela empresa Dow Corning Silício do Brasil.

Materiais líquidos: água doce e aditivos fornecidos pela empresa Schlumberger que são: antiespumante (D175), dispersante (D080) e retardador de pega (D801).

Devido ao sigilo industrial as frações dos compostos não são fornecidas neste artigo.

\subsection{AVALIAÇÃO DA ENERGIA DE MISTURA}

Para os ensaios de energia de mistura foi utilizado o misturador Chandler, modelo 30-60 (Figura 3a). Neste misturador a energia de mistura era controlada através do tempo e velocidade de cisalhamento.

$\mathrm{O}$ material sólido era pesado e homogeneizado. Os compostos líquidos eram postos no misturador e a mistura sólida era adicionada gradualmente durante 60 segundos, sob agitação de 4000 RPM. Após isso, era dado inicio a contagem do tempo e a rotação era fixada em valor específico.

Foram feitas análises nos tempos de 420, 540, 900 e 1200 segundos, nas rotações de 4000, 5000 e 6000 RPM. Em cada conjunto tempo-rotação foi feito ensaio reológico no viscosímetro FANN 35A (Figura 3b) e a determinação da massa específica, na balança de lama (Figura 3c). A temperatura também foi registrada.

(a)

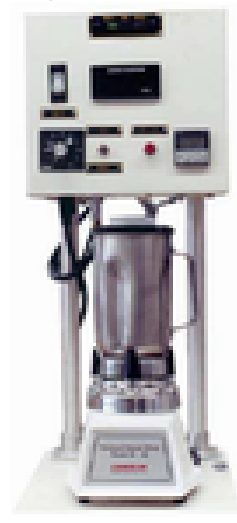

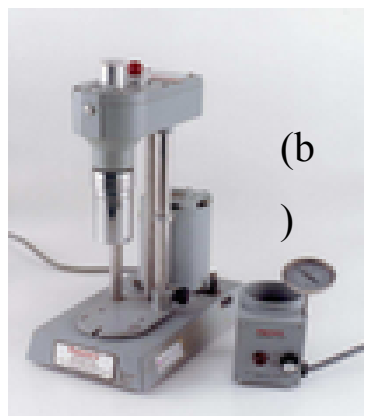




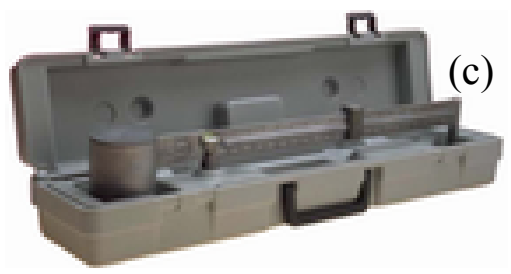

Figura 3. (a) Misturador Chandler, modelo 30-60; (b) Viscosímetro FannVG Meter, Modelo 35A; (c) Balança de Lama, Fann.

\subsection{AVALIAÇÃO DA PERDA DE CARGA}

Assim como no experimento de bancada, os materiais sólidos eram misturados previamente, nesse caso em um misturador $\mathrm{Y}$. O material líquido era adicionado ao tanque e a mistura sólida gradualmente adicionada sob a fração líquida. A pasta produzida era mantida em circulação em um sistema fechado de escoamento (Figuras 4 e 5).

Os experimentos foram feitos nas temperaturas de 15,25 e $60^{\circ} \mathrm{C}$. Amostras da pasta eram coletadas para ensaios reológicos em viscosímetro FANN 35A.

A unidade experimental consiste de um loop fechado de tubos de ferro galvanizado; um tanque de 250 litros com sistema de agitação e aquecimento; medidor de vazão do tipo Coriolis da Metroval (modelo RHM40), transdutor de pressão diferencial da Smar (modelo LD301D), bomba helicoidal da Weatherford de $25 \mathrm{HP}$ e um resfriador Friotec (modelo TF-15AR) para o controle da temperatura.

$O$ trecho experimental foi composto por quatro dutos com as seguintes geometrias: circular 1", anular I (2" e 1 1/2"), anular II (1 1/2" e 1 ") e anular III (1 1/4" e 3/4"). Nesses trechos foram feitas as tomadas de diferença de pressão por meio do transdutor.
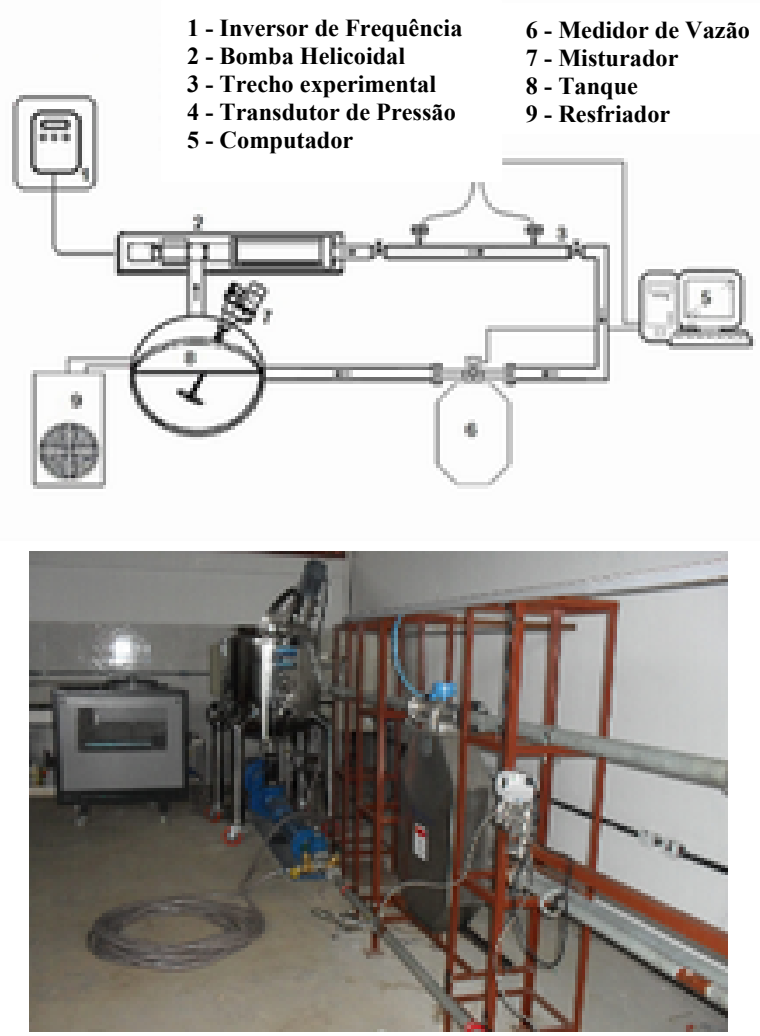

Figuras 4 e 5. Esquema e foto da unidade experimental de escoamento de fluidos.

De posse dos dados de diferença de pressão, vazão e dados reológicos obtidos, foi possível a avaliação de correlações pertinentes à previsão da perda de carga. Na Figura 6 um fluxograma com a metodologia utilizada.

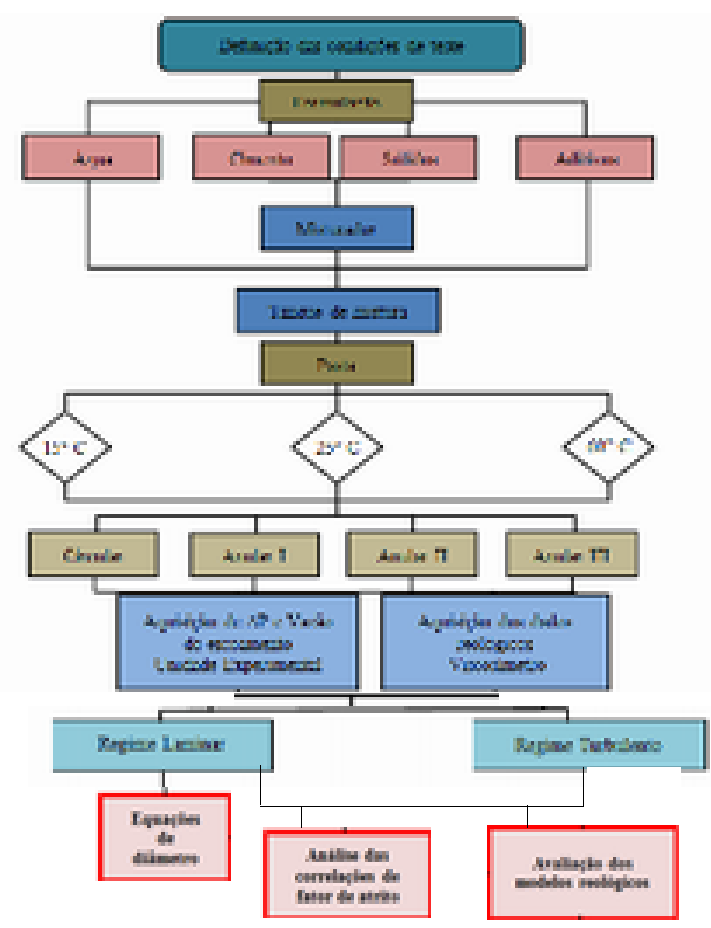

Figura 6. Fluxograma da metodologia do teste das pastas de cimento. 


\section{RESULTADOS E DISCUSSÃO}

Neste tópico, os resultados da análise das equações pertinentes à previsão da perda de carga da pasta de cimento são apresentados. Além disto, também é apresentada a avaliação dos resultados obtidos do efeito da energia de mistura sobre a quebra das microesferas e suas consequências sobre propriedades físicoquímica da pasta de cimento.

\subsection{AVALIAÇÃO DOS MODELOS REOLÓGICOS}

Com os dados de deformação angular e taxa de cisalhamento fornecidos pelo viscosímetro foi possível o cálculo dos parâmetros reológicos dos modelos power law, Bingham e Herschel-Buckley.

$\mathrm{Na}$ Tabela 1 são apresentadas as médias dos parâmetros reológicos, bem como o coeficiente de correlação ao quadrado $\left(\mathrm{R}^{2}\right)$ obtido em cada modelo.

Tabela 1. Parâmetros reológicos médios obtidos a 15,25 e $60^{\circ} \mathrm{C}$.

\begin{tabular}{|c|c|c|c|c|}
\hline \multirow[b]{2}{*}{ DUTOS } & \multirow[b]{2}{*}{$\mathrm{T}(\stackrel{\circ}{ } \mathrm{C})$} & \multicolumn{3}{|c|}{ COEFICIENTE DE CORRELÇÃO } \\
\hline & & $\begin{array}{c}\text { Power } \\
\text { Law }\end{array}$ & Bingham & $\begin{array}{c}\text { Herschel- } \\
\text { Buckley }\end{array}$ \\
\hline \multirow{3}{*}{ Circular } & 15 & 0,9716 & 0,9973 & 0,9998 \\
\hline & 25 & 0,9673 & 0,9978 & 0,9999 \\
\hline & 60 & 0,9570 & 0,9970 & 0,9998 \\
\hline \multirow{3}{*}{ Anular I } & 15 & 0,9712 & 0,9967 & 0,9998 \\
\hline & 25 & 0,8319 & 0,9943 & 0,9949 \\
\hline & 60 & 0,9693 & 0,9936 & 0,9965 \\
\hline \multirow{3}{*}{ Anular II } & 15 & 0,9802 & 0,9965 & 0,9992 \\
\hline & 25 & 0,9738 & 0,9930 & 0,9999 \\
\hline & 60 & 0,9582 & 0,9967 & 0,9993 \\
\hline \multirow{3}{*}{ Anular III } & 15 & 0,9906 & 0,9936 & 0,9998 \\
\hline & 25 & 0,9815 & 0,9925 & 0,9998 \\
\hline & 60 & 0,9636 & 0,9962 & 0,9997 \\
\hline \multirow{3}{*}{ MÉDIA } & 15 & 0,9811 & 0,9954 & 0,9998 \\
\hline & 25 & 0,9387 & 0,9944 & 0,9986 \\
\hline & 60 & 0,9620 & 0,9959 & 0,9988 \\
\hline
\end{tabular}

Como pode ser visto na tabela anterior, o modelo de Herschel-Buckley é o que apresenta o melhor coeficiente de correlação ao quadrado. Posteriormente o de Bingham, apresentando bons resultados e depois o de power law.

\subsection{DIÂMETRO HIDRÁULICO}

As avaliações de diâmetro hidráulico foram feitas com os dados obtidos em regime laminar.

Os erros percentuais médios absolutos das equações do diâmetro hidráulico foram calculados utilizando a Equação 33.

Erro $=\left|\frac{D H_{E_{x p}}-D H_{T e \text { or }}}{D H_{\text {Exp }}}\right| 100$

$\mathrm{Na}$ Tabela 2 são apresentados os erros percentuais médios absolutos das equações dos diâmetros hidráulicos aplicadas nos anulares para os três modelos reológicos e nas temperaturas analisadas

Através da Tabela 2 pode-se concluir que a equação de diâmetro hidráulico $\mathrm{DH}_{1}$ obteve, de maneira geral, melhores resultados nas três temperaturas e nos três anulares, utilizando o modelo reológico power law.

A equação do diâmetro hidráulico $\mathrm{DH}_{3}$ obteve pontos satisfatórios na temperatura de $60^{\circ} \mathrm{C}$, utilizando o modelo reológico power law. A equação do diâmetro hidráulico $\mathrm{DH}_{2}$ obteve pontos satisfatórios unicamente na temperatura de $60^{\circ} \mathrm{C}$, no duto anular I e utilizando os três modelos reológicos analisados. 
Tabela 2. Erros percentuais médios absolutos dos diâmetros hidráulicos.

\begin{tabular}{|c|c|c|c|c|c|c|c|c|c|c|}
\hline \multirow{2}{*}{ ANULAR } & \multirow{2}{*}{$\mathrm{T}(\stackrel{\circ}{ } \mathrm{C})$} & \multicolumn{3}{|c|}{ POWER LAW } & \multicolumn{3}{|c|}{ BINGHAM } & \multicolumn{3}{|c|}{ HERSCHEL - BUCKLEY } \\
\hline & & $\mathrm{DH} 1$ & $\mathrm{DH} 2$ & $\mathrm{DH} 3$ & $\mathrm{DH} 1$ & $\mathrm{DH} 2$ & $\mathrm{DH} 3$ & $\mathrm{DH} 1$ & $\mathrm{DH} 2$ & $\mathrm{DH} 3$ \\
\hline \multirow[t]{2}{*}{1} & 25 & 30,04 & 50,32 & 42,63 & 101,52 & 35,34 & 49,81 & 104,13 & 37,34 & 51,57 \\
\hline & 60 & 14,52 & 18,34 & 15,07 & 77,12 & 21,06 & 33,05 & 45,91 & 12,42 & 15,65 \\
\hline \multirow[t]{3}{*}{ II } & 25 & 29,05 & 65,89 & 57,98 & 114,32 & 49,12 & 33,16 & 86,09 & 29,36 & 65,40 \\
\hline & 60 & 19,55 & 32,39 & 20,51 & 119,98 & 48,49 & 94,20 & 46,22 & 54,54 & 41,91 \\
\hline & 15 & 19,47 & 61,41 & 48,00 & 99,24 & 53,27 & 35,15 & 55,76 & 55,61 & 39,21 \\
\hline III & 15 & 19,47 & 61,41 & 48,00 & 99,24 & 53,27 & 35,15 & 55,76 & 55,61 & 39,21 \\
\hline \multirow[t]{2}{*}{ MÉDIA } & 25 & 26,83 & 58,59 & 48,86 & 109,70 & 44,48 & 37,49 & 85,91 & 39,45 & 50,29 \\
\hline & 60 & 17,31 & 25,98 & 17,75 & 103,16 & 37,86 & 75,22 & 59,39 & 30,87 & 42,55 \\
\hline
\end{tabular}




\subsection{ANÁLISE DAS EQUAÇÕES DE FATOR DE ATRITO}

A análise das equações de fator de atrito foi feita através de gráficos $\log -\log$ de Fator de atrito versus Reynolds, onde foram plotados os dados experimentais e os dados teóricos.

Foram obtidos gráficos para todos os casos estudados. Para ilustrar a análise realizada, na Figura 7 é apresentado um resultado típico que foi obtido no teste realizado a $25^{\circ} \mathrm{C}$ usando o modelo de Herschel-Buckley.

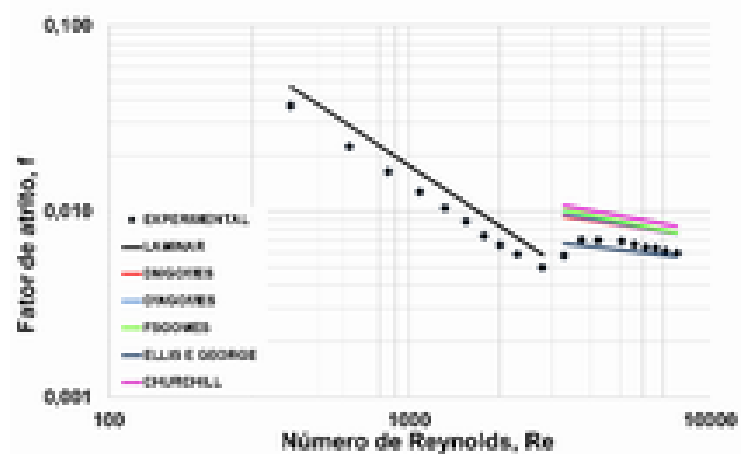

Figura 7. Gráfico de fator de atrito versus Reynolds - duto circular de 1" modelo de Herschel-Buckley, $25^{\circ} \mathrm{C}$.

Os mesmos gráficos foram feitos para os três modelos reológicos nas três temperaturas e nos 4 dutos estudados. Os erros percentuais absolutos foram calculados através da Equação 34.

$$
\text { Erro }=\left|\frac{f_{\text {Exp }}-f_{T \text { eor }}}{f_{\text {Exp }}}\right| 100
$$

Os valores médios desses erros foram agrupados nas Tabelas de 3 a 5 . Nestas tabelas, para fins ilustrativos, os erros percentuais menores que $25 \%$ são destacados.

De acordo com as Tabelas de 3 a 5, no regime laminar, o modelo cuja correlação de fator de atrito obteve menores erros percentuais foi o power law.

Gomes, no modelo da potência e Darby e Melson (1981) no modelo de Bingham.

Nas geometrias anulares, a utilização da correlação $\mathrm{DH}_{1}$, de maneira geral, trouxe melhores resultados quando associadas às correlações indicadas anteriormente.

Tabela 3. Erros percentuais médios absolutos das correlações de fator de atrito a $15^{\circ} \mathrm{C}$.

\begin{tabular}{|c|c|c|c|c|c|c|c|c|c|c|c|}
\hline \multirow{2}{*}{$\begin{array}{l}\text { MODELOS } \\
\text { REOLÓGICOS }\end{array}$} & \multirow{2}{*}{$\begin{array}{l}\text { CORRELAÇÃO DE } \\
\text { FAROR DE ATRITO }\end{array}$} & \multirow{2}{*}{ CIRCULAR } & \multicolumn{3}{|c|}{ ANULAR I } & \multicolumn{3}{|c|}{ ANULAR II } & \multicolumn{3}{|c|}{ ANULAR III } \\
\hline & & & DH1 & DH2 & DH3 & DH1 & $\mathrm{DH} 2$ & DH3 & DH1 & $\mathrm{DH} 2$ & $\mathrm{DH} 3$ \\
\hline \multirow{6}{*}{ POWER LAW } & Laminar & 15,8 & 35,4 & 11,5 & 10,7 & 18,1 & 31,5 & 21,0 & 19,5 & 61,4 & 48,0 \\
\hline & DM Gomes & 11,4 & - & - & - & - & - & - & 8,4 & 86,9 & 84,4 \\
\hline & OW Gomes & 16,5 & - & - & - & - & - & - & 7,0 & 87,5 & 85,1 \\
\hline & FS Gomes & 15,4 & - & - & - & - & - & - & 6,8 & 85,8 & 83,0 \\
\hline & Ellis e George & 13,8 & - & - & - & - & - & - & 9,5 & 87,8 & 85,2 \\
\hline & Churchill & 29,1 & - & - & - & - & - & - & 37,6 & 81,4 & 75,0 \\
\hline \multirow{8}{*}{ BINGHAM } & Laminar & 33,8 & 29,0 & 51,7 & 46,6 & 118,7 & 46,4 & 92,9 & 99,2 & 53,3 & 34,5 \\
\hline & Darby e Melson & 16,0 & - & - & - & - & - & - & 8,9 & 90,0 & 88,0 \\
\hline & Tomita & 41,5 & - & - & - & - & - & - & 66,2 & 79,4 & 74,5 \\
\hline & Darby et. al. & 33,0 & - & - & - & - & - & - & 28,3 & 92,1 & 90,6 \\
\hline & Ellis e George & 6,0 & - & - & - & - & - & - & 19,6 & 84,7 & 80,8 \\
\hline & Churchill & 61,4 & - & - & - & - & - & - & 52,5 & 66,6 & 53,0 \\
\hline & Laminar & 27,4 & 31,5 & 53,9 & 49,2 & 73,3 & 18,1 & 22,6 & 55,8 & 55,6 & 39,2 \\
\hline & DM Gomes & 35,5 & - & - & - & - & - & - & 36,3 & 84,1 & 80,7 \\
\hline
\end{tabular}




\begin{tabular}{ccccccccccc} 
OW Gomes & 36,9 & - & - & - & - & - & - & 36,8 & 83,6 & 80,3 \\
FS Gomes & 42,7 & - & - & - & - & - & - & 46,9 & 81,3 & 77,4 \\
Ellis e George & 7,9 & - & - & - & - & - & - & 6,9 & 86,1 & 82,8 \\
Churchill & 55,3 & - & - & - & - & - & - & 38,3 & 73,6 & 63,6 \\
\hline
\end{tabular}

Tabela 4. Erros percentuais médios absolutos das correlações de fator de atrito a $25^{\circ} \mathrm{C}$.

\begin{tabular}{|c|c|c|c|c|c|c|c|c|c|c|c|}
\hline \multirow{2}{*}{$\begin{array}{l}\text { MODELOS } \\
\text { REOLÓGICOS }\end{array}$} & \multirow{2}{*}{$\begin{array}{l}\text { CORRELAÇÃO DE } \\
\text { FAROR DE ATRITO }\end{array}$} & \multirow{2}{*}{ CIRCULAR } & \multicolumn{3}{|c|}{ ANULAR I } & \multicolumn{3}{|c|}{ ANULAR II } & \multicolumn{3}{|c|}{ ANULAR III } \\
\hline & & & $\mathrm{DH}_{1}$ & $\mathrm{DH} 2$ & $\mathrm{DH} 3$ & $\mathrm{DH} 1$ & $\mathrm{DH} 2$ & $\mathrm{DH} 3$ & $\mathrm{DH} 1$ & $\mathrm{DH} 2$ & $\mathrm{DH} 3$ \\
\hline \multirow{6}{*}{ POWER LAW } & Laminar & 16,3 & 30,0 & 50,3 & 42,6 & 29,1 & 65,9 & 58,0 & 21,4 & 59,6 & 46,0 \\
\hline & DM Gomes & 12,8 & 31,7 & 43,8 & 41,9 & 10,7 & 85,9 & 83,5 & 4,4 & 87,1 & 84,6 \\
\hline & OW Gomes & 19,0 & 45,4 & 55,2 & 53,6 & 1,7 & 87,0 & 84,8 & 7,8 & 88,0 & 85,7 \\
\hline & FS Gomes & 18,4 & 43,2 & 53,7 & 51,8 & 3,9 & 85,4 & 82,9 & 4,1 & 86,5 & 83,8 \\
\hline & Ellis e George & 14,0 & 14,1 & 24,2 & 22,2 & 6,8 & 86,2 & 83,7 & 5,3 & 87,5 & 84,9 \\
\hline & Churchill & 22,3 & 22,5 & 5,9 & 9,1 & 55,7 & 82,8 & 78,7 & 40,5 & 83,4 & 77,8 \\
\hline \multirow{6}{*}{ BINGHAM } & Laminar & 28,5 & 101,5 & 35,3 & 49,8 & 114,3 & 49,1 & 33,2 & 113,2 & 49,0 & 28,7 \\
\hline & Darby e Melson & 18,5 & 17,5 & 6,9 & 6,3 & 16,9 & 87,4 & 85,1 & 4,3 & 89,1 & 87,0 \\
\hline & Tomita & 31,7 & 104,3 & 59,5 & 67,4 & 111,8 & 74,1 & 68,6 & 78,7 & 78,0 & 72,7 \\
\hline & Darby et. al. & 36,0 & 9,1 & 26,9 & 24,2 & 8,3 & 90,1 & 88,3 & 22,1 & 91,5 & 89,8 \\
\hline & Ellis e George & 5,8 & 47,2 & 15,6 & 21,1 & 51,9 & 80,8 & 76,5 & 28,5 & 83,8 & 79,6 \\
\hline & Churchill & 51,5 & 82,7 & 52,1 & 57,1 & 87,9 & 58,3 & 44,8 & 55,3 & 66,7 & 52,7 \\
\hline \multirow{6}{*}{$\begin{array}{l}\text { HERSCHEL - } \\
\text { BUCKLEY }\end{array}$} & Laminar & 25,9 & 104,1 & 37,3 & 51,6 & 46,2 & 54,5 & 41,9 & 67,5 & 51,6 & 33,9 \\
\hline & DM Gomes & 30,1 & 27,2 & 53,6 & 63,3 & 59,1 & 80,2 & 76,7 & 42,6 & 83,2 & 79,6 \\
\hline & OW Gomes & 31,6 & 42,1 & 58,6 & 68,8 & 57,3 & 80,0 & 76,4 & 41,7 & 82,9 & 79,4 \\
\hline & FS Gomes & 35,3 & 40,7 & 67,8 & 79,0 & 67,2 & 77,3 & 73,0 & 51,1 & 80,7 & 76,5 \\
\hline & Ellis e George & 8,0 & 7,2 & 12,0 & 17,2 & 25,4 & 83,0 & 79,6 & 10,3 & 85,5 & 82,1 \\
\hline & Churchill & 45,1 & 27,9 & 54,7 & 59,0 & 75,1 & 68,7 & 59,8 & 56,8 & 74,7 & 64,9 \\
\hline
\end{tabular}

Tabela 5. Erros percentuais médios absolutos das correlações de fator de atrito a $60^{\circ} \mathrm{C}$.

\begin{tabular}{|c|c|c|c|c|c|c|c|c|c|c|c|}
\hline \multirow{2}{*}{$\begin{array}{l}\text { MODELOS } \\
\text { REOLÓGICOS }\end{array}$} & \multirow{2}{*}{$\begin{array}{l}\text { CORRELAÇÃO DE } \\
\text { FAROR DE ATRITO }\end{array}$} & \multirow{2}{*}{ CIRCULAR } & \multicolumn{3}{|c|}{ ANULAR I } & \multicolumn{3}{|c|}{ ANULAR II } & \multicolumn{3}{|c|}{ ANULAR III } \\
\hline & & & $\mathrm{DH} 1$ & $\mathrm{DH} 2$ & DH3 & $\mathrm{DH} 1$ & $\mathrm{DH} 2$ & $\mathrm{DH} 3$ & $\mathrm{DH} 1$ & $\mathrm{DH} 2$ & $\mathrm{DH} 3$ \\
\hline \multirow{6}{*}{ POWER LAW } & Laminar & 16,3 & 14,5 & 18,3 & 15,1 & 19,6 & 32,4 & 20,5 & 17,9 & 27,2 & 17,7 \\
\hline & DM Gomes & 7,3 & 14,8 & 16,6 & 13,6 & 16,6 & 7,8 & 8,1 & 8,8 & 13,8 & 5,5 \\
\hline & OW Gomes & 16,7 & 12,3 & 25,7 & 21,4 & 4,3 & 17,6 & 3,6 & 4,2 & 23,1 & 7,7 \\
\hline & FS Gomes & 16,8 & 9,5 & 22,2 & 17,5 & 7,0 & 15,6 & 1,3 & 2,1 & 21,4 & 5,2 \\
\hline & Ellis e George & 7,7 & 16,5 & 17,0 & 14,5 & 16,2 & 7,7 & 8,5 & 10,3 & 12,8 & 8,0 \\
\hline & Churchill & 32,3 & 49,7 & 25,9 & 31,3 & 62,8 & 28,7 & 50,9 & 52,0 & 20,6 & 45,4 \\
\hline \multirow{6}{*}{ BINGHAM } & Laminar & 40,0 & 77,1 & 21,1 & 33,1 & 120,0 & 48,5 & 94,2 & 112,4 & 44,0 & 98,4 \\
\hline & Darby e Melson & 9,2 & 24,9 & 10,0 & 11,6 & 25,5 & 4,3 & 16,4 & 16,0 & 10,5 & 11,4 \\
\hline & Tomita & 41,2 & 86,0 & 43,1 & 52,7 & 118,4 & 67,0 & 101,1 & 100,6 & 52,6 & 88,8 \\
\hline & Darby et. al. & 28,3 & 10,0 & 24,4 & 19,7 & 3,6 & 23,4 & 8,7 & 9,1 & 29,8 & 14,6 \\
\hline & Ellis e George & 11,2 & 61,4 & 24,3 & 32,0 & 57,6 & 21,2 & 45,4 & 45,1 & 11,5 & 36,7 \\
\hline & Churchill & 61,1 & 111,6 & 47,8 & 59,2 & 93,3 & 63,6 & 84,8 & 90,7 & 56,1 & 84,1 \\
\hline \multirow{4}{*}{$\begin{array}{l}\text { HERSCHEL - } \\
\text { BUCKLEY }\end{array}$} & Laminar & 38,2 & 45,9 & 12,4 & 15,7 & 86,1 & 29,4 & 65,4 & 86,0 & 25,7 & 70,1 \\
\hline & DM Gomes & 41,0 & 57,8 & 24,3 & 31,9 & 100,9 & 56,3 & 86,1 & 82,1 & 44,6 & 76,1 \\
\hline & OW Gomes & 41,3 & 56,2 & 22,7 & 30,3 & 105,0 & 59,2 & 89,8 & 85,6 & 47,0 & 79,3 \\
\hline & FS Gomes & 42,4 & 63,8 & 27,7 & 35,9 & 116,8 & 67,3 & 100,2 & 96,6 & 53,8 & 88,9 \\
\hline
\end{tabular}




\begin{tabular}{ccccccccccc} 
Ellis e George & 9,7 & 23,7 & 7,1 & 8,8 & 46,1 & 13,6 & 35,2 & 33,6 & 6,7 & 28,8 \\
Churchill & 54,5 & 74,1 & 44,9 & 51,4 & 105,1 & 64,9 & 94,4 & 88,5 & 53,4 & 83,5 \\
\hline
\end{tabular}

\subsection{ENERGIA DE MISTURA}

$\mathrm{Na}$ Tabela 6 são apresentados os dados de massa específica obtidos em experimentos de bancada, realizados no misturador Chandler, modelo 3060. Nesta tabela são mostrados ainda a velocidade de rotação e o tempo de exposição ao cisalhamento, informações pertinentes ao cálculo da energia de mistura.

Tabelo 6. Dados experimentais para o cálculo da energia de mistura.

\begin{tabular}{cccc}
\hline $\boldsymbol{\omega}(\mathbf{R P M})$ & Tempo (s) & $\mathbf{E}(\mathbf{J})$ & $\boldsymbol{\rho}$ exp. $\left(\mathbf{K g} / \mathbf{m}^{\mathbf{3}}\right)$ \\
\hline \multirow{4}{*}{4000} & 420 & 6988 & 1490 \\
& 540 & 8984 & 1500 \\
& 900 & 14973 & 1510 \\
& 1200 & 19964 & 1520 \\
\hline \multirow{4}{*}{5000} & 420 & 10918 & 1510 \\
& 540 & 14037 & 1520 \\
& 900 & 23396 & 1540 \\
6000 & 1200 & 31194 & 1560 \\
& 420 & 15722 & 1535 \\
& 540 & 20214 & 1550 \\
& 900 & 33690 & 1570 \\
& 1200 & 44920 & 1590 \\
\hline
\end{tabular}

A partir dos dados da Tabela 6 , foi construído o gráfico de massa específica versus energia de mistura apresentada na Figura 8.

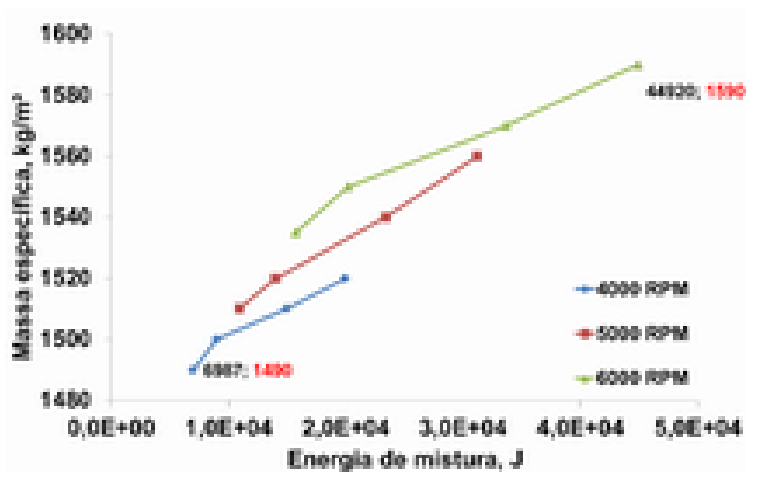

Figura 8. Massa específica versus energia de mistura.

É possível observar na Tabela 6 e na Figura 8 que o aumento da massa específica ocorre em decorrência do crescimento da energia de mistura. Este fato reafirma o que foi mostrado em Miranda (2008), onde a autora relaciona a quebra das microesferas com o consequente aumento da massa específica e energia de mistura. Além disto, o aumento na taxa de cisalhamento intensifica a quebra das microesferas causando uma variação mais significativa da massa específica, para um mesmo valor de energia de mistura aplicada.

Nas Figuras de 9 a 11 são apresentados os dados de taxa de deformação e tensão cisalhante obtidos nos ensaios reológicos. Estes ensaios foram realizados no viscosímetro FANN $35 \mathrm{~A}$ nas amostras preparadas em rotações de 4000, 5000 e $6000 \mathrm{rpm}$.

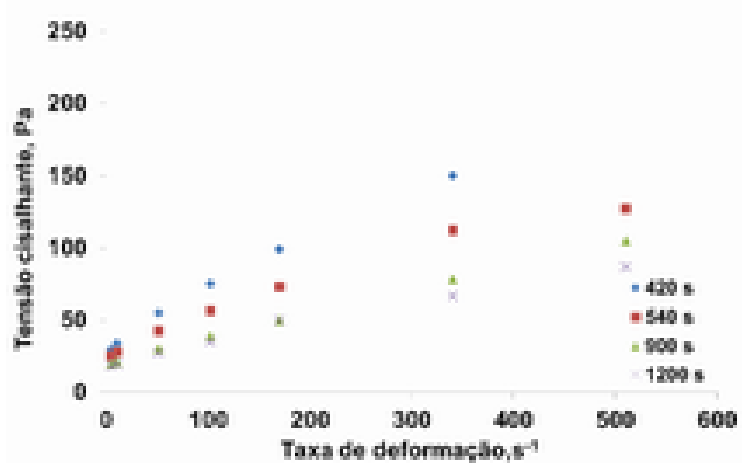

Figura 9. Tensão cisalhante versus taxa de deformação para rotação de $4000 \mathrm{rpm}$.

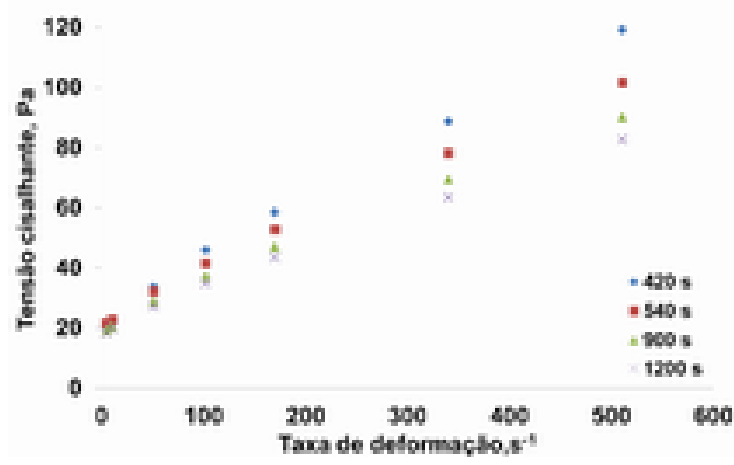

Figura 10. Tensão cisalhante versus taxa de deformação para rotação de $5000 \mathrm{rpm}$. 


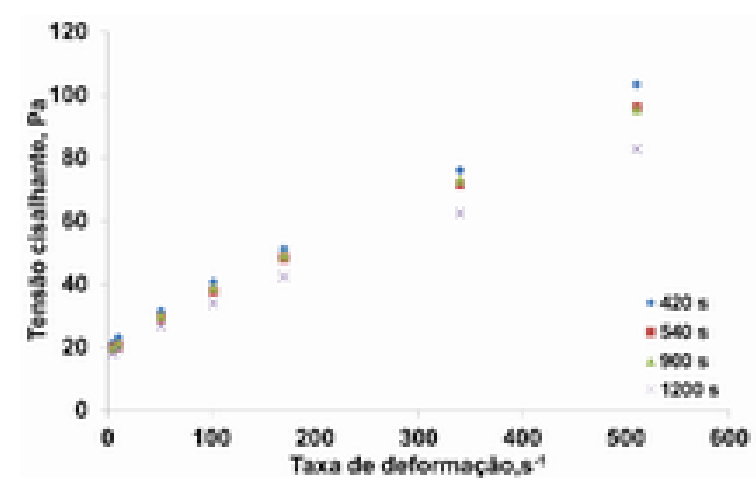

Figura 11. Tensão cisalhante versus taxa de deformação para rotação de $6000 \mathrm{rpm}$.

A partir das Figuras de 9 a 11, pode-se observar que o aumento da energia de mistura, proporcionado pela velocidade e pelo tempo de rotação, diminui o diferencial de tensão cisalhante. Esse comportamento ocorre devido à completa desfloculação sofrida pela pasta e, concomitante, aumento na quebra das microesferas.

$\mathrm{Na}$ Figura 12, a viscosidade aparente, calculada com dados da taxa de deformação de $511 \mathrm{~s}^{-1}$, é relacionada com a energia de mistura fornecida no preparo das pastas.

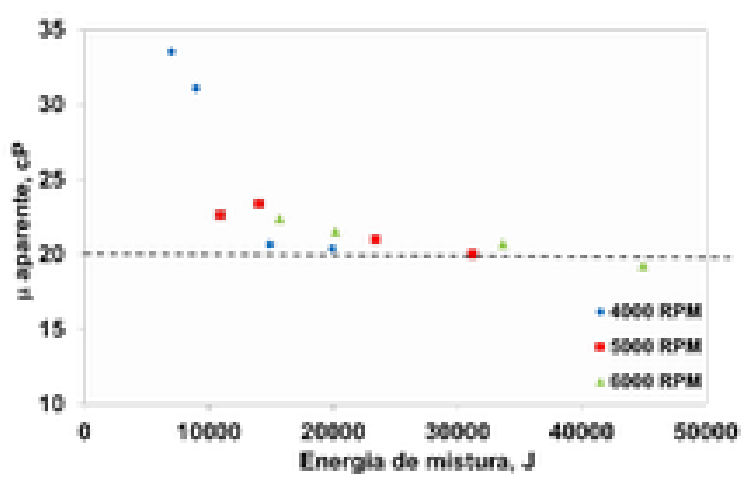

Figura 12. Viscosidade aparente versus energia de mistura por rotação e sob taxa de deformação de $511 \mathrm{~s}^{-1}$.

$\mathrm{Na}$ Figura 12, observa-se que a viscosidade plástica diminui com $\mathrm{o}$ aumento continuo da energia de mistura. A partir de $2,0.10^{4} \mathrm{~J}$ é possível notar que há uma tendência de estabilização da viscosidade aparente da pasta de cimento. Esta estabilização provavelmente está relacionada a uma completa quebra das microesferas. Esta quebra indica um ponto crítico deste sistema já que a partir de então, a microesfera perde sua funcionalidade de reduzir a massa específica da pasta. Esse efeito, ocorrendo durante o processo de campo, acarretaria em alterações contínuas nas pressões de bombeio proporcionando um risco adicional a operação.

\section{CONCLUSÃO}

Foi analisada em um loop de escoamento a perda de carga de uma pasta de cimento que continha em sua composição microesfera cerâmica, um aditivo estendedor. Além disso, o efeito da energia de mistura sobre a quebra das microesfera foi acompanhado em experimentos de bancada.

Com relação ao escoamento da pasta de cimento, pode-se concluir que o modelo reológico Herschel-Buckley obteve o índice de correlação mais próximo da unidade. Independente da temperatura analisada, este modelo foi o que melhor descreveu o comportamento reológico da pasta.

Quanto às correlações de fator de atrito, no regime laminar, o modelo power law apresentou os menores erros em todas as temperaturas analisadas. No regime turbulento, a correlação de Ellis e George (1977) obteve os menores desvios no escoamento da pasta. Esta correlação também se destaca pelos bons resultados nos três modelos reológicos. As equações propostas por Gomes (1987), também foram satisfatórias utilizando o modelo power law.

Em relação à energia de mistura, pode-se comprovar que seu aumento intensifica a quebra das microesferas. Além da energia de mistura, percebe-se que o aumento na taxa de cisalhamento da pasta amplifica o efeito de quebra das microesferas corroborando para $o$ aumento da massa específica. $\mathrm{O}$ aumento da energia de mistura causou ainda uma redução da viscosidade aparente do fluido.

Os resultados dos testes de energia de mistura apontam para um fator relevante sobre o preparo das pastas em laboratório e sua produção nas sondas de perfuração. As alterações nas propriedades da pasta a diferentes taxas de cisalhamento podem prejudicar o dimensionamento da pressão de bombeio 
e trazer risco a estabilidade hidráulica e mecânica do poço.

\section{REFERÊNCIAS}

BIRD, R. B.; STEWART, W. E.; LIGHTFOOT, E. N., 2004. Fenômeno de Transporte. $2^{\mathrm{a}}$ edição, Editora LTC.

BOURGOYNE Jr., A. T., MILLHEIM, K. K., CHENEVERT, M. E., and YOUNG Jr, F. S., 1991. Applied drilling engineering. Second printing, Society of petroleum engineers, Richardson, Texas.

CAMPOS, G.; SIMÃO A. C.; TEIXEIRA C. K.; CONCEIÇÃO F. C. A., 2002. Curso básico sobre cimentação primária (Apostila interna). CENPES/ PETROBRAS.

CHURCHILL, S. W., 1977. Friction factor equation spans all fluid flow regimes. Chem. Eng. 7.

DARBY, R. AND MELSON, J., 1981. How to predict the friction factor for the flow of Bingham plastics. Chem Eng. vol. 88 , no. 26, pp. 59-61.

DARBY, R., MUN, R., BOGER D. V.,1992. Predict Friction Loss in Slurry Pipes. Chemical Engineering. v.9, p.116.

ELLIS, R. C. E GEORGE, D. S., 1977. Practical interpretation on theology, annular displacing torces. How to avoid by passing mud during primary cementing. World Oil, 64-9.

GOMES, F. J. A. D., 1987. Hidráulica, fluidos de potência: racionalização do uso do fator de fricção. Encontro Técnico sobre Informática na Perfuração, CAPER/87, Módulo 7, Salvador, Brasil.

MIRANDA, C. R., 2008. Pastas de cimento de alta compacidade para poços de petróleo - processo de formulação, propriedades reológicas, resistência mecânica e química. Tese de doutorado, Instituto Militar de Engenharia do Rio de Janeiro, Rio de Janeiro, Brasil.
NELSON, E.; GUILLOT, D., 1990. Well Cementing. 2nd. ed. Texas: Schlumberger.

PARAISO, E. C. H., 2011. Estudo do escoamento de pasta de cimento em dutos circulares $e$ anulares. Dissertação de mestrado, Universidade Federal Rural do Rio de Janeiro, Rio de Janeiro, Brasil.

THOMAS, J. E.; TRIGGIA, A. A.; CORREIA, C. A.; FILHO, C. V.; XAVIER, J. A. D.; MACHADO, J. C. V.; FILHO, J. E. S.; PAULA, J. L.; ROSSI, N. C. M.; PITOMBO, N. E. S.; GOUVEIA, P. C. V. M.; CARVALHO, R. S. E BARRAGEM, R. V., 2001. Fundamentos de Engenharia de Petróleo. Editor Interciência. Rio de Janeiro.

TOMITA, Y., 1959. A study on nonNewtonian flow in pipe lines. Bulletin JSME, v. 2, n. 5, p. 10-16.

\section{AGRADECIMENTOS}

Os autores agradecem ao Centro de Pesquisa da Petrobras (CENPES), Coordenação de Aperfeiçoamento de Pessoal de Nível Superior (CAPES) e Conselho Nacional de Desenvolvimento Científico e Tecnológico (CNPq), pelo apoio técnico e financeiro. 